\title{
The use of SD-OCT in the differential diagnosis of dots, spots and other white retinal lesions
}

This article was published in the following Dove Press journal:

Eye and Brain

25 October 2011

Number of times this article has been viewed

\author{
Elena Zaharova' \\ Jerome Sherman ${ }^{1-3}$ \\ 'State University of New York's State \\ College of Optometry, University \\ Eye Center, New York, NY, USA; \\ ${ }^{2}$ SUNY Eye Institute, New York, NY, \\ USA; ${ }^{3} \mathrm{New}$ York Eye Institute and \\ Laser Center, New York, NY, USA
}

Correspondence: Elena Zaharova 4 Bristol Road, Marblehead, MA 0I945, USA

Tel +l 78I 5882137

Email zaharovaod@gmail.com
Purpose: To demonstrate the utility of a retinal imaging technique using spectral domain optical coherence tomography (SD-OCT) for creating a B-scan layer-by-layer analysis to aid in the differential diagnosis of various retinal dots, spots, and other white lesions.

Design: Review.

Methods: A retrospective review of imaging studies performed with SD-OCT (Topcon, 3DOCT2000, Oakland, NJ) at SUNY State College of Optometry.

Results: B-scan layer-by-layer analysis and unique SD-OCT reflectivity patterns of the following retinal white lesions are reviewed in the order of their retinal layer localization: myelinated nerve fiber layer, cotton wool spot, exudates, edema residues, drusen, fundus albipunctatus, Stargardt disease, Bietti crystalline dystrophy, punctate inner choroidopathy (PIC), presumed ocular histoplasmosis syndrome (POHS), post-photocoagulation chorioretinal scarring, and osseous choristoma.

Conclusion: The reviewed images demonstrate the utility of SD-OCT in the identification of the unique characteristics of the presented retinal pathologies. SD-OCT is ideal for retinal layer localization of lesions, thus enhancing the differential diagnosis of retinal dots, spots, and other white lesions. Even though true pathognomonic patterns are rare, highly suggestive findings of certain retinal abnormalities often facilitate immediate recognition and diagnosis.

Keywords: SD-OCT, photoreceptor integrity line, retinal pigment epithelium, white dot syndrome, retinal pathology, imaging

\section{Introduction}

Optical coherence tomography (OCT) has revolutionized our approach to retinal disease identification and diagnosis. It is a noninvasive imaging procedure of ocular structures that employs nonionizing optical radiation. ${ }^{1,2}$ Recent work in the field of ophthalmic OCT has demonstrated that spectral or Fourier detection methods (SD-OCT) enable OCT imaging with significantly improved speed, resolution, and sensitivity, as opposed to time domain OCT (TD-OCT). ${ }^{3-6}$ SD-OCT is capable of obtaining 27,000-40,000 A-scans/second with an axial resolution of 3.5-6 $\mu \mathrm{m},{ }^{1,7}$ as opposed to an acquisition speed of $400 \mathrm{~A}$-scans/second, as in TD-OCT. ${ }^{2}$ Thus, SD-OCT imaging results in considerable improvement in image acquisition speed and resolution, minimizing the well-known limitations of TD-OCT, such as limited retinal nerve fiber layer (RNFL) resolution, operator error, and increased motion artifacts. ${ }^{1,2}$ The unique capability of SD-OCT technology is to provide a layer-by-layer analysis of the retina with a resolution highly superior to contact lens fundus ophthalmoscopy and/or fundus photography. Clinically detectable drusen by ophthalmoscopy, for example, 
is $30 \mu \mathrm{m}^{8}$ and resolution of fundus photography is $25 \mu \mathrm{m},{ }^{9}$ whereas axial resolution of SD-OCT has been reported to be $3.5-6 \mu \mathrm{m} .{ }^{1,7}$ The high sensitivity of this instrument provides entirely new imaging capabilities and, therefore, has significant impact on diagnosis and decision making.

$\mathrm{SD}-\mathrm{OCT}$ is often referred to in literature as "optical biopsy method" due to its extraordinary ability to provide information on ocular subsurface structures with retinal layerby-layer analysis, otherwise obtainable only by histology. ${ }^{3}$ The interface between different retinal layers is determined by changes in reflective properties between the tissues. ${ }^{1}$ One of the most important new SD-OCT developments secondary to the higher resolution is the recognition and clinical importance of the photoreceptor integrity line (PIL), ${ }^{10}$ also referred to as the IS/OS line. PIL represents the junction between the inner (IS) and outer (OS) segments of the photoreceptors (Figure 1). Even though it is barely visible on histological sections, PIL is highly reflective with SD-OCT imaging. Recent histological studies have shown that the second hyperreflective outer retinal OCT band, currently attributed to IS/ OS junction, is likely due to mitochondria packing geometry and the organelle's high refractive index (1.4). ${ }^{11}$ Costa et al suggested that the inner layer, which is identical to the PIL reported in this study, could not be visualized with OCT imaging due to either a missing or disrupted PIL in the eyes of patients with outer retinal disorders. ${ }^{12}$ Moreover, studies have shown that the integrity of the PIL is associated with visual function. ${ }^{13,14}$ Shin et al have recently reported that the length of a disrupted PIL on SD-OCT in patients with retinal vein occlusion is closely associated, if not predictive, of visual outcome. ${ }^{14}$ High reflectivity and integrity of the
PIL has been found to be independent of age and low scan sensitivities. ${ }^{10}$ In normal eyes, PIL is as visible as the retinal pigment epithelium (RPE). If the PIL disappears while the RPE is still visible, it is likely that the PIL is abnormal in this region of the retina.

The current uses of SD-OCT are extensive and include retinal nerve fibre layer (RNFL) analysis, vitreomacular interface evaluation, and macular abnormalities. This study will explore SD-OCT characteristics of some common retinal and choroidal pathologies that present as white lesions: myelinated nerve fiber layer, cotton wool spot, exudates, edema residues, drusen, fundus albipunctatus, Stargardt disease, Bietti crystalline dystrophy, punctate inner choroidopathy (PIC), presumed ocular histoplasmosis syndrome (POHS), post-photocoagulation chorioretinal scarring, and osseous choristoma. In this study, the above retinal white dots, spots, and other lesions are categorized based on the retinal layer where they are thought to be found. The pathologies that affect the inner retina are myelinated nerve fiber layer and cotton wool spot. Retinal lesions that are typically localized to the outer retina are exudates and edema residues. Retinal pigment epithelium (RPE) abnormalities are further subdivided into sub-RPE deposits such as in drusen and fundus albipunctatus and RPE migration and/or atrophy cases such as in Stargardt disease, Bietti crystalline dystrophy, PIC, POHS, and post-photocoagulation chorioretinal scarring. In this study, B-scan analysis of retinal layers and unique SD-OCT reflectivity patterns of the white deposits in each of the above conditions are presented, stressing the importance of SD-OCT imaging, and the evaluation of PIL and RPE integrity in the differential diagnosis.

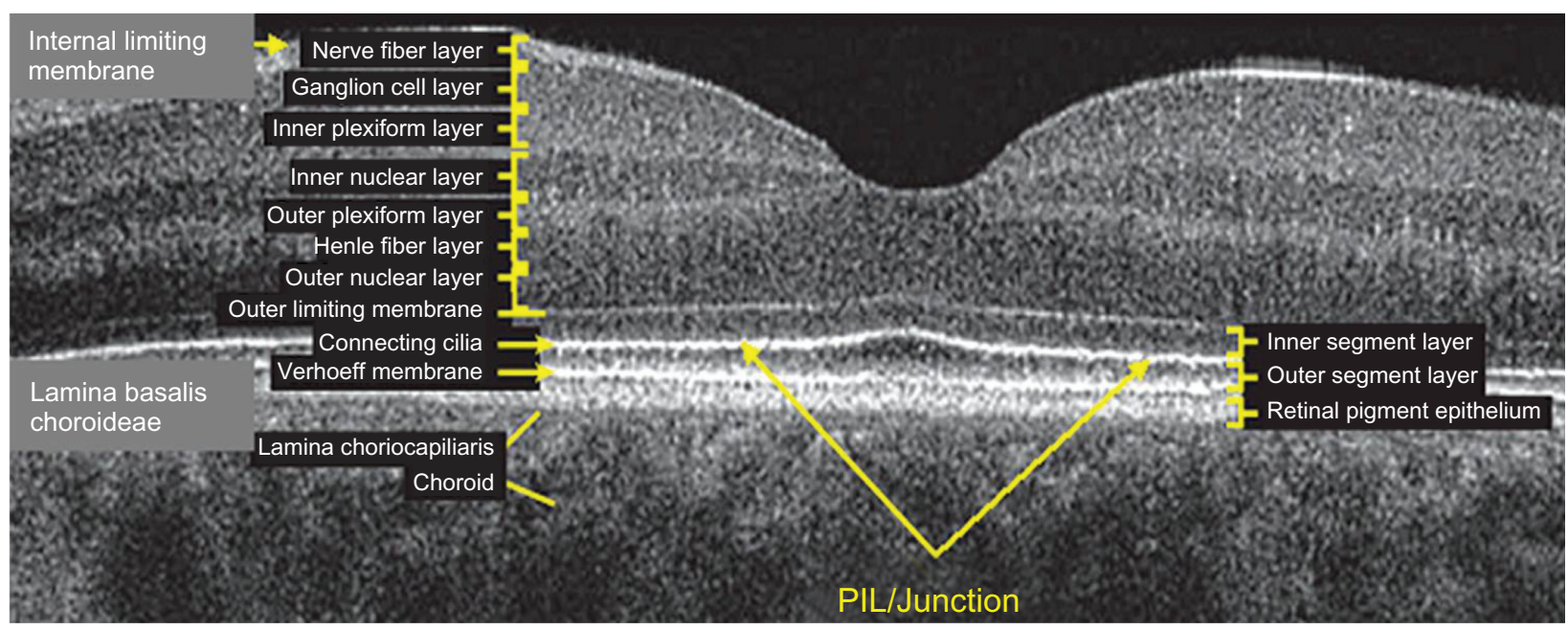

Figure I Layer-by-layer B-scan SD-OCT display of normal retina. Abbreviation: SD-OCT, spectral domain optical coherence tomography. 


\section{Methods}

This study presents a retrospective review of imaging studies performed with SD-OCT (Topcon, 3DOCT-2000, Oakland, $\mathrm{NJ}$ ) at the SUNY State College of Optometry. The B-scan image of the PIC case included in this study was taken with Spectralis OCT (Heidelberg Engineering, Carlsbad, CA).

\section{Results}

\section{Inner retinal lesions}

Myelinated retinal nerve fiber layer (MRNF)

MRNF is reported to be present in approximately $1 \%$ of the population. Histological studies show the presence of oligodendrocyte-like glial cells and the surrounding myelination that is thought to be due to developmental anomaly or acquired insult. ${ }^{15}$ Generally, during embryogenesis the process of myelination should stop at the level of the lamina cribrosa due to the dense aggregation of astrocytes that acts as a barrier. ${ }^{15}$ Abnormal migration of oligodendrocyte-like cells into the retina results in MRNF formation and is thought to happen either prior to the development of the barrier function of lamina cribrosa or due to the abnormal dislocation of the glial cells as a result of a temporary loss of the barrier function of the lamina cribrosa. ${ }^{15}$ The condition is usually asymptomatic; however, wide-spread myelination is associated with increased degree of axial myopia and hypoplastic disc in the affected eye. ${ }^{15}$ Usually no treatment is indicated unless neovascularization or vitreous hemorrhage complications arise. ${ }^{15}$ Clinically, MRNF appears as white or gray-white striated patches with feathery edges (Figure 2). Myelination can be localized to the vascular arcades, be contiguous with the disc, or be separate from it. The size depends on the severity of developmental anomaly ranging from small 1-2DD $(1500-3000 \mu \mathrm{m})$ to large, extending into the posterior pole and mid-periphery.

\section{B-scan SD-OCT reflectivity pattern}

Figure 2 shows hyper-reflective thickened RNFL. The increased thickness of the RNFL completely blocks the view of underlying structures. The increase in thickness of MRNF closer to the disc, the part of retina where RNFL is the thickest, should be noted.

\section{Cotton wool spots (CWS)}

CWSs develop secondary to focal ischemia associated with occlusion of precapillary arterial flow. ${ }^{16-19}$ Blood flow abnormality, rather than a simple infarct is thought to cause an ischemic event. ${ }^{19}$ There are numerous retinal vascular and systemic diseases associated with formation of cotton

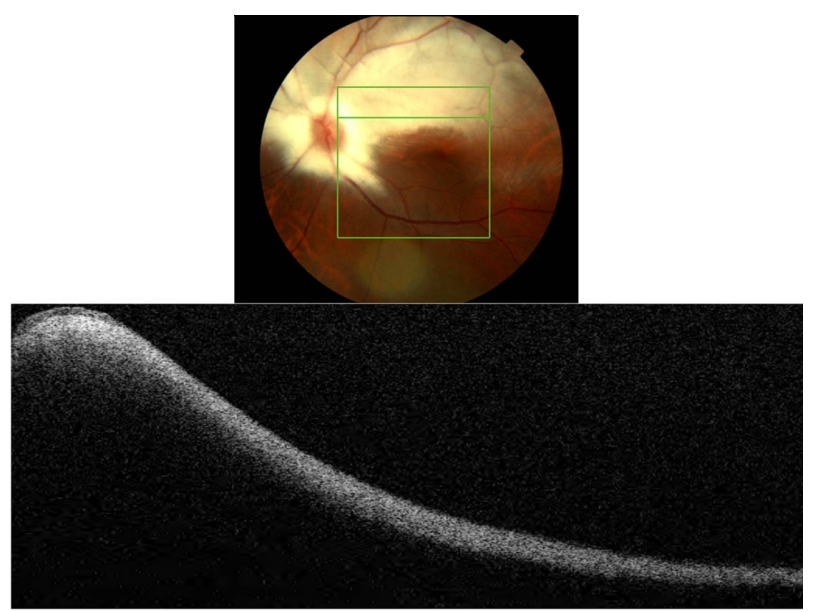

Figure 2 Fundus photograph (top) showing MRNF; the B-scan line on the fundus photograph has the same width as the B-scan SD-OCT image (bottom); SD-OCT image demonstrates thickened RNFL due to MRNF, thickness of which increases closer to the optic nerve head.

Abbreviations: SD-OCT, spectral domain optical coherence tomography; RNFL, retinal nerve fiber layer; MRNF, myelinated retinal nerve fiber layer.

wool spots. ${ }^{16-18}$ There can be an ischemic etiology, embolic, infectious, tractional, traumatic, or simply idiopathic. ${ }^{16} \mathrm{CWSs}$ are comprised of swollen ganglion cell axons that expand as much as 10 -fold (to some $5-15 \mu \mathrm{m}$ in diameter) due to focal ischemic events. ${ }^{19}$ Light microscopy studies have revealed the presence of cytoid bodies within a swollen axon. ${ }^{16-19}$ Cytoid bodies form due to the accumulation of intracytoplasmic organelles that were interrupted in their normal axoplasmic flow. ${ }^{16-18}$ Clinically, CWSs appear as slightly elevated white or yellow-white lesions with cloud-like or fluffy borders (Figure 3) ${ }^{16-18} \mathrm{CWSs}$ are typically localized to nerve fiber layer and develop at the border of large ischemic areas. The typical size of CWSs is $<0.25 \mathrm{DD}(<400 \mu \mathrm{m})$. They usually resolve within 5-12 weeks, but may persist for 1-2 years in diabetic patients. ${ }^{17,18}$

\section{B-scan SD-OCT reflectivity pattern}

Figure 3 shows a hyper-reflective thickened area within the RNFL that appears as a nodular shape rather than continuously thickened RNFL. The retinal layers underneath the CWS are "masked" by the thickened nerve fibers.

\section{Outer retinal lesions}

\section{Hard exudates}

There are numerous retinal vascular diseases associated with hard exudates, including diabetic retinopathy, hypertensive retinopathy, retinal venous obstruction, retinal arterial macroaneurysm, radiation retinopathy, Coat's disease, and capillary hemangioma. ${ }^{20}$ Exudates are frequently associated 


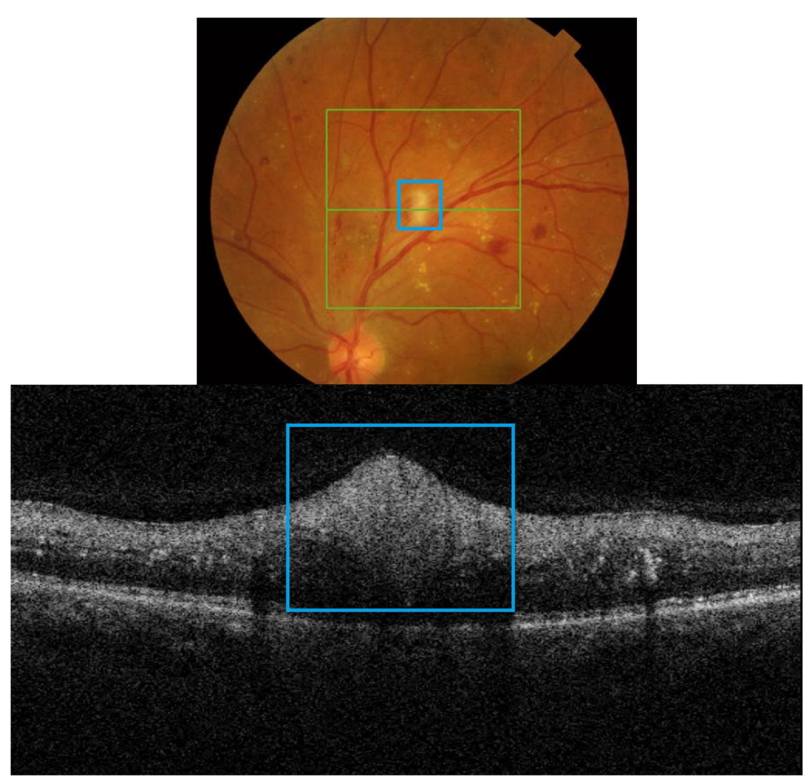

Figure 3 Fundus photograph (top) showing CWS (blue rectangle) in the left eye of a patient with diabetic retinopathy; the B-scan line on the fundus photograph has the same width as the B-scan SD-OCT image (bottom); SD-OCT image demonstrates thickened RNFL in CWS (blue rectangle).

Abbreviations: SD-OCT, spectral domain optical coherence tomography; RNFL, retinal nerve fiber layer; CWS, cotton wool spot.

with choroidal neovascular membrane (CNVM) formation in the wet form of age-related macular degeneration (ARMD). The pathophysiology of exudates is described by chronic localized leakage of plasma as a result of vasodilation. ${ }^{20}$ This causes a deposition of lipid, which can be visualized clinically as waxy white-yellow lesions with distinct margins (Figure 4). Exudates are mainly composed of lipoprotein and lipid-filled macrophages. The deposition is thought to occur

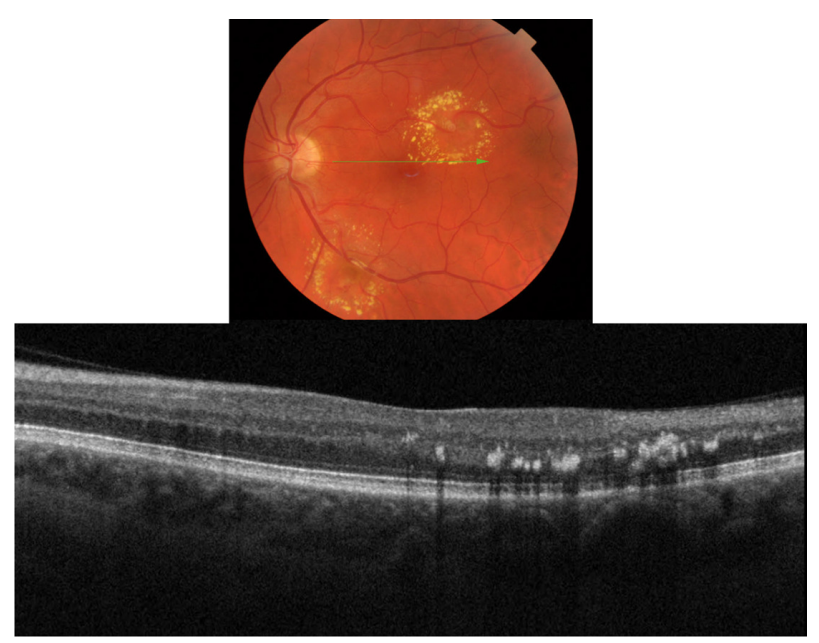

Figure 4 Fundus photograph (top) showing a circinate ring of exudates in the left eye of a patient with macroaneurysm; the B-scan line on the fundus photograph has the same width as the B-scan SD-OCT image (bottom); SD-OCT image demonstrates exudate deposits at the level of outer plexiform layer (OPL).

Abbreviation: SD-OCT, spectral domain optical coherence tomography. in the outer plexiform layer (OPL) of the retina. The size of exudates is usually small, ranging between $15-55 \mu \mathrm{m}$, with those smaller than $25 \mu \mathrm{m}$ invisible to ophthalmoscopy and fundus photography. ${ }^{20}$ The lipid deposits typically develop at the junction of normal and edematous retina, arranging themselves as clumps and rings, which are commonly termed a circinate ring in diabetic retinopathy and a macular star in hypertensive retinopathy. It generally takes months to years for exudates to reabsorb. ${ }^{20}$

\section{B-scan SD-OCT reflectivity pattern}

Our SD-OCT images of patients with exudates confirm the location of the deposits in and around OPL retinal layer (Figure 4). Deposits present as hyper-reflective minute dots at the level of OPL. One hundred percent of the time the differential diagnosis between drusen and exudates can be made by layer localization of the deposits in question (Figure 5). Figure 5 shows fundus photography (top) and its corresponding SD-OCT image (bottom) demonstrating a B-scan through the two different types of deposit: exudates (blue square) and drusen (red arrow).

Figure 6 illustrates the example of superior resolution of SD-OCT in comparison to fundus photography. Nearly all of the hard exudates in this horizontal section are invisible to ophthalmoscopy but easily detected with SD-OCT. By reviewing all 128 horizontal sections in the $6 \times 6 \mathrm{~mm}$ box, 405 exudates were detected with an SD-OCT; whereas only 20 were visualized with ophthalmoscopy, a 20 -fold sensitivity improvement.

\section{Subretinal deposits/edema residues/retinal precipitates}

Edema residues, also referred to as subretinal deposits or retinal precipitates, are commonly seen in patients with central serous chorioretinopathy (CSC); $86 \%$ of patients with CSC were reported to present with white deposits in the area of retinal detachment. ${ }^{9}$ Edema residues begin as minute yellowish deposits of granular material that are believed to contain photoreceptor outer segment debris. ${ }^{9}$ Later these deposits coalesce together into dense confluent patches of amorphous extravascular fibrinous material. ${ }^{9}$ Clinically, edema residues are similar in appearance to hard exudates (Figure 7), presenting as waxy white-yellow lesions with distinct margins. ${ }^{21}$ Deposits are variable in size ranging from small $(10-20 \mu \mathrm{m})$ to large $(200-300 \mu \mathrm{m}){ }^{9}$ Generally, edema residues are located in front of the RPE layer and they are highly confined to the detached area of retina. ${ }^{9}$ 


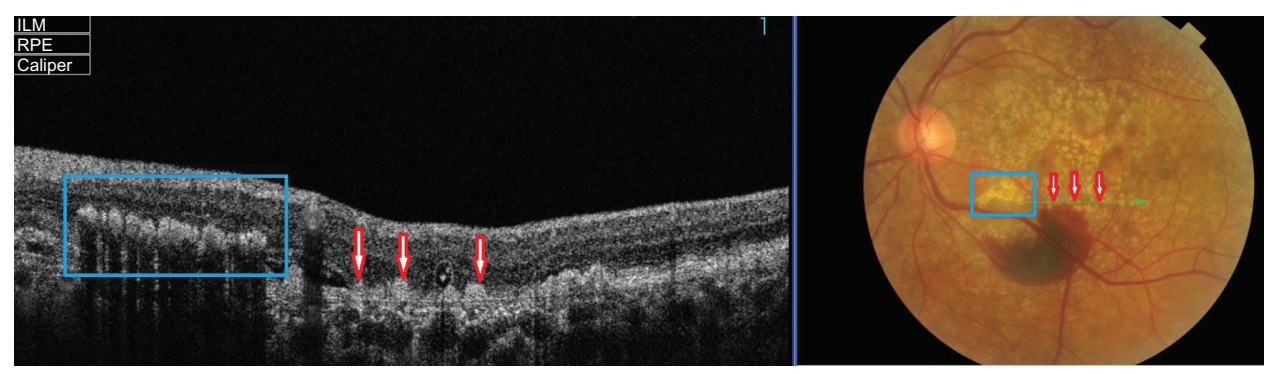

Figure 5 Fundus photograph (right) showing two different types of deposit: exudates (blue rectangle) and drusen (red arrow) in the left eye of a patient with wet ARMD; the B-scan line on the fundus photograph has the same width as the B-scan SD-OCT image (left).

Abbreviations: ARMD, age-related macular degeneration; SD-OCT, spectral domain optical coherence tomography.

\section{B-scan SD-OCT reflectivity pattern}

Figure 8 demonstrates edema residues that appear as hyperreflective deposits (red arrows) within the area of retinal detachment. SD-OCT images confirm the location of subretinal deposits to the photoreceptor outer segment layer. These subretinal precipitates are virtually pathognomonic of CSC.

\section{Retinal pigment epithelium (RPE) lesions Sub-RPE deposits}

Drusen

Drusen deposits are commonly associated with ARMD and dominant drusen. ARMD is known to be the leading cause of irreversible legal blindness in people over 60 years of age. ${ }^{22}$ It is characterized by the progressive loss of central vision due to degeneration, dysfunction, and subsequent death of photoreceptor cells. ${ }^{23}$ Drusen-induced RPE degeneration has been hypothesized to account for the loss of photoreceptor cells. ${ }^{22,23}$ The largest single component of drusen is lipid, mainly esterified and unesterified cholesterol and phosphatidylcholine. ${ }^{24}$ There are also other components such as polysaccharides, glycosaminoglycans, and protein molecules that

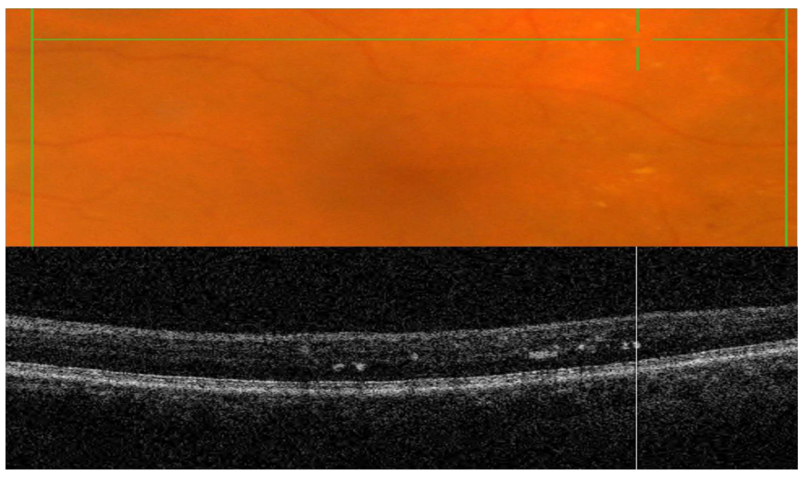

Figure 6 Cross-section of a scanned fundus image (top); the B-scan line on the fundus photograph has the same width as the B-scan SD-OCT image (bottom). Abbreviations: SD-OCT, spectral domain optical coherence tomography; ARMD, age-related macular degeneration. mediate proinflammatory and immune processes ${ }^{22,24}$ Cellular components within drusenoid deposits have been found to be RPE debris, lipofuscin, and melanin. ${ }^{22}$ All types and sizes of drusen, including subclinical drusen smaller than $63 \mu \mathrm{m}$, have been reported to result in photoreceptor abnormalities, not affecting horizontal, amacrine, bipolar, and ganglion cells. ${ }^{23}$

Three types of drusen have been recently described: hard, subretinal drusenoid deposits, and soft..$^{24}$ In this study, hard drusen will be further subdivided into cuticular and infiltrative based on the SD-OCT reflective characteristics. Clinically, hard drusen appears as multiple densely packed small yellow-white nodular deposits of lipid-rich material (Figures 9 and 10). Typically it is $50-75 \mu \mathrm{m}$ in size. The location of the deposits distinguishes hard drusen from subretinal drusenoid deposits.

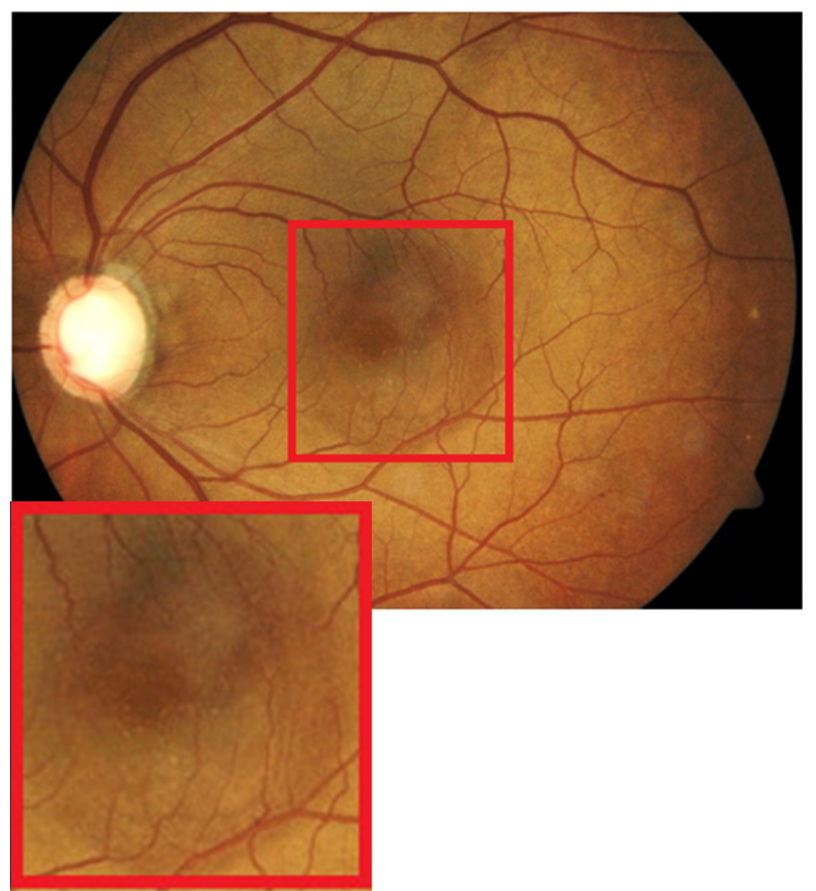

Figure 7 Fundus photograph of the left eye showing edema residues inside the area of CSC (enlarged).

Abbreviation: CSC, central serous chorioretinopathy. 


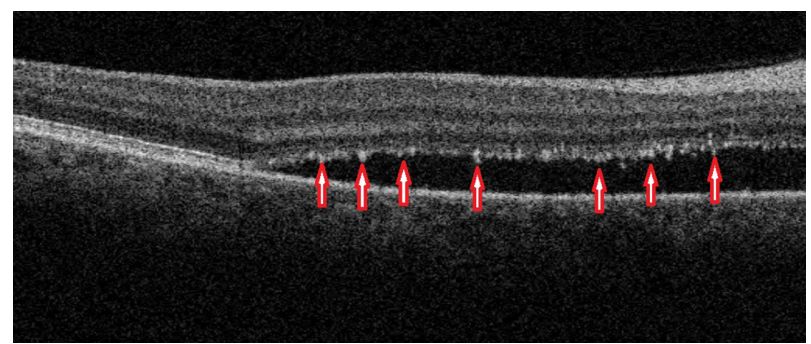

Figure 8 B-scan SD-OCT image demonstrating edema residues confined to the level of photoreceptor outer segment layer in CSC.

Abbreviations: SD-OCT, spectral domain optical coherence tomography; CSC, central serous chorioretinopathy.

Subretinal drusenoid deposits are similar in appearance to hard drusen and are similar in size (50-75 $\mu \mathrm{m})$ (Figure 11). However, in comparison to hard drusen, as Spaide and Curcio $^{24}$ reported, drusenoid-material is located above rather than below the RPE layer. Soft drusen can be distinguished more easily from the other types of drusen based on their larger size (63-1000 $\mu \mathrm{m}$ in diameter). ${ }^{24}$ It appears as yellowwhite, dome-shaped, lipid-rich deposits with softer borders (Figure 12). Similar to the hard drusen, soft drusen deposits are located between the RPE and Bruch's membrane.

\section{B-scan SD-OCT reflectivity pattern}

Cuticular hard drusen appear as discrete dome-shaped elevations of RPE layer due to the deposits external to the RPE basement membrane and solid in appearance (Figure 9).

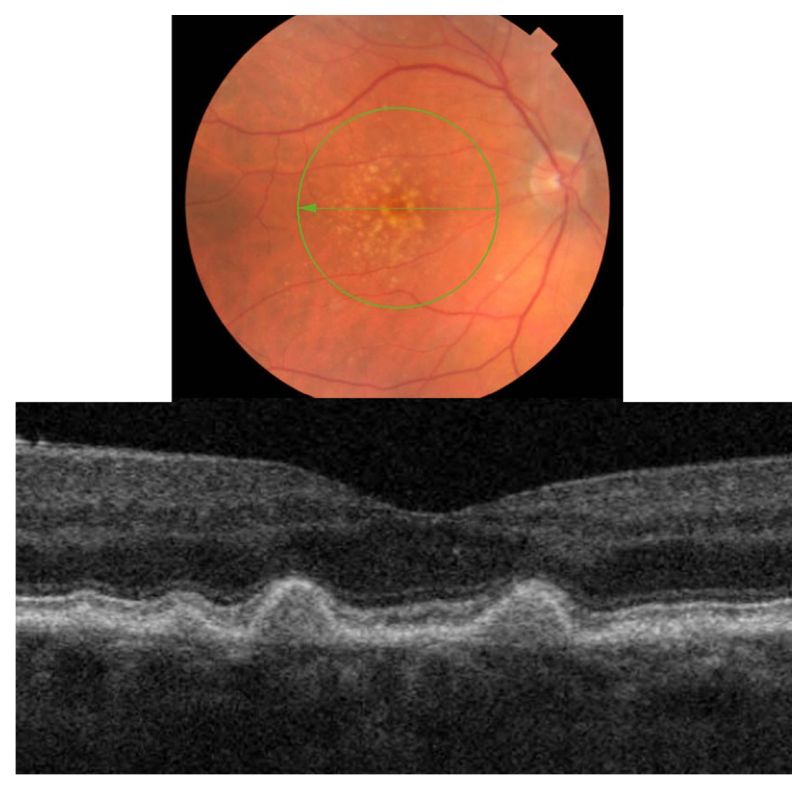

Figure 9 Fundus photograph (top) showing hard cuticular drusen in the right eye of a patient with ARMD; the B-scan line on the fundus photograph has the same width as the B-scan SD-OCT image (bottom) which demonstrates the appearance of hard cuticular drusen.

Abbreviations: SD-OCT, spectral domain optical coherence tomography; ARMD, age-related macular degeneration.

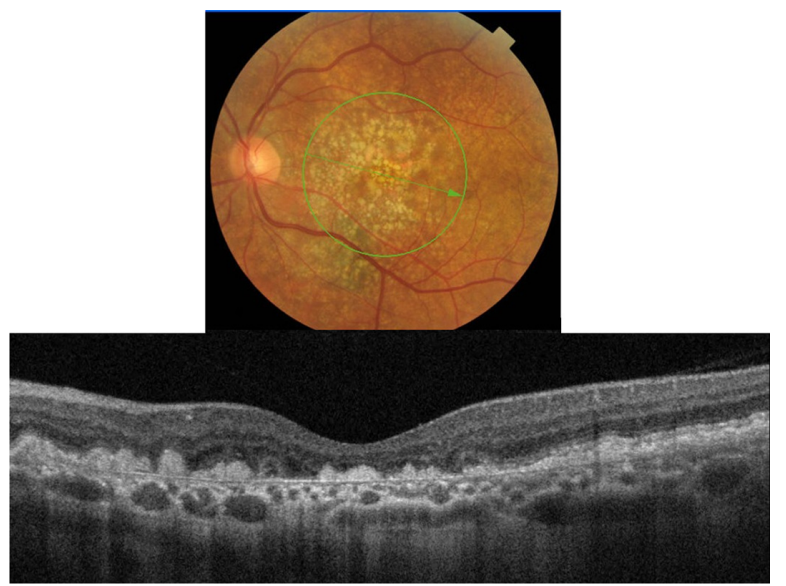

Figure I 0 Fundus photograph (top) showing hard infiltrative drusen in the left eye of a patient with ARMD; the B-scan line on the fundus photograph has the same width as the B-scan SD-OCT image (bottom) which demonstrates the appearance of hard infiltrative drusen.

Abbreviations: SD-OCT, spectral domain optical coherence tomography; ARMD, age-related macular degeneration.

It can be clearly visualized with SD-OCT imaging that the RPE layer is intact and elevated with sub-RPE deposits beneath it. Elevations have about the same height-to-width ratio. Another important distinguishing feature of cuticular hard drusen is the presence of PIL that sits on top of the domeshaped structures. Even though it is thinner than in unaffected parts of retina, the PIL is generally present and intact.

Infiltrative hard drusen, on the other hand, appear drastically different with SD-OCT (Figure 10). Multiple discrete deposits infiltrate through the RPE and PIL with disruption of both layers.

Subretinal drusenoid deposits are conical in shape and have a more punctuate appearance. SD-OCT images show with great resolution the fact that deposits are localized between the RPE and the PIL layer (Figure 11). PIL is intact and slightly elevated with an intact outer limiting membrane sitting on top of it. In contrast to hard drusen, however, the RPE layer is not elevated because the deposits are sitting on top of it, rather than underneath.

Soft drusen exhibit a classical appearance with sub-RPE deposits that result in a pronounced elevation of the RPE (Figure 12). SD-OCT reveals elevated mounds or dome-shaped structures with less reflective material inside as compared with the solid-appearance of a hard drusen. Large elevated drusen are typically associated with a compromised PIL above the deposits, which can be clearly visualized with the SD-OCT B-scan. Another important feature of soft drusen is that in some cases it is possible to visualize Bruch's membrane, which appears as a thin reflective layer that lies flat at the base of a soft drusen. Generally Bruch's membrane is masked by the 


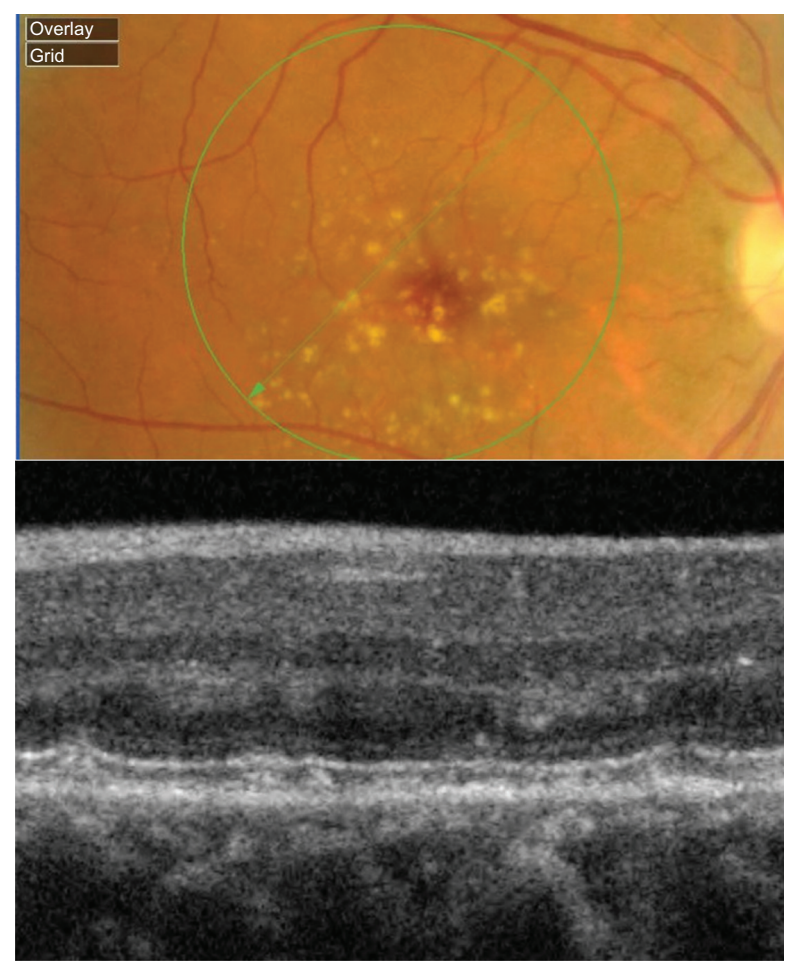

Figure I I Fundus photograph (top) showing subretinal drusenoid deposits in the right eye of a patient with ARMD; the B-scan line on the fundus photograph has the same width as the B-scan SD-OCT image (bottom) which demonstrates the appearance of subretinal drusenoid deposits.

Abbreviations: SD-OCT, spectral domain optical coherence tomography; ARMD, age-related macular degeneration.

RPE shadow; however, when the RPE is compromised, it is possible to localize the presence of Bruch's membrane.

\section{Geographic atrophy}

Analysis of fundus photography images of the right and left eyes of the patient who presented with subretinal drusenoid

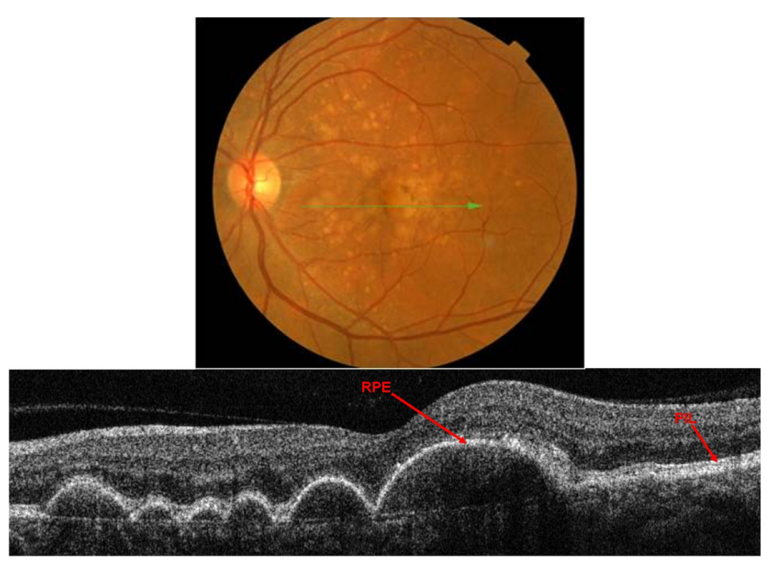

Figure 12 Fundus photograph (top) showing soft drusen in the left eye of a patient with ARMD; the B-scan line on the fundus photograph has the same width as the B-scan SD-OCT image (bottom) which demonstrates the appearance of soft drusen. Abbreviations: SD-OCT, spectral domain optical coherence tomography; ARMD, age-related macular degeneration. deposits revealed one area of deposits that appeared different from the rest (Figure 13). SD-OCT imaging was done through the region of interest (marked by a blue arrow). As Figure 13 shows, B-scan reveals missing PIL and the external limiting membrane, along with the easily visualized Bruch's membrane, the presentation consistent with geographic atrophy. Drusenoid deposits, marked by red arrows, surround the area of geographic atrophy.

\section{Fundus albipunctatus (fundus albi)}

Fundus albi (FA) is a rare form of congenital stationary night blindness (CSNB) characterized by minute white dots and a delayed course of dark adaptation. ${ }^{25}$ The condition is stationary and nonprogressive. ${ }^{25}$ A combination of FA and cone dystrophy (CD) has been reported in several studies. ${ }^{27}$ $\mathrm{CD}$ and FA have been linked by a mutation in the 11-cis retinol dehydrogenase (RDH5) gene with autosomal recessive inheritance reported in at least 6 families. ${ }^{25-27}$ RDH5 is expressed predominantly in the RPE cells and plays a role in the visual cycle, ${ }^{26,27}$ which can explain the symptoms of night blindness. The composition of the white deposits is unknown, but they have been reported to be localized to the level of the RPE. ${ }^{27}$

Clinically, FA presents as a large number of discrete monotonous subretinal white or pale-yellow deposits in the perimacular area and mid-periphery (Figures 14 and 15). The deposits are usually best visualized with a green-separation filter which localizes deposits to the retinal rather than the choroidal layer. The white dots usually fade slowly over decades.

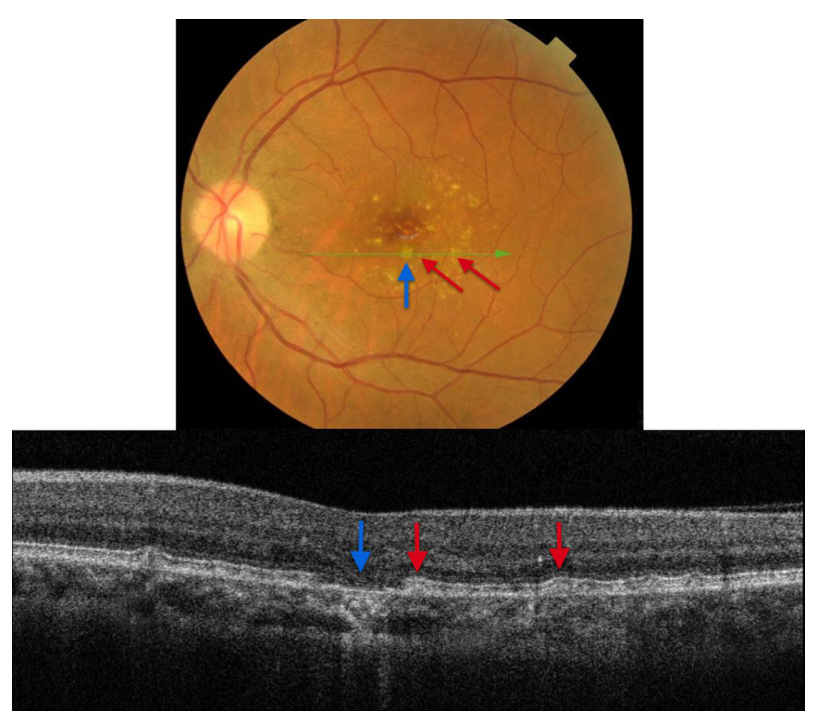

Figure 13 The B-scan line on the fundus photograph (top) has the same width as the B-scan SD-OCT image (bottom) demonstrating one area of geographic atrophy (blue arrow) surrounded by multiple subretinal drusenoid deposits (red arrows). Abbreviation: SD-OCT, spectral domain optical coherence tomography. 


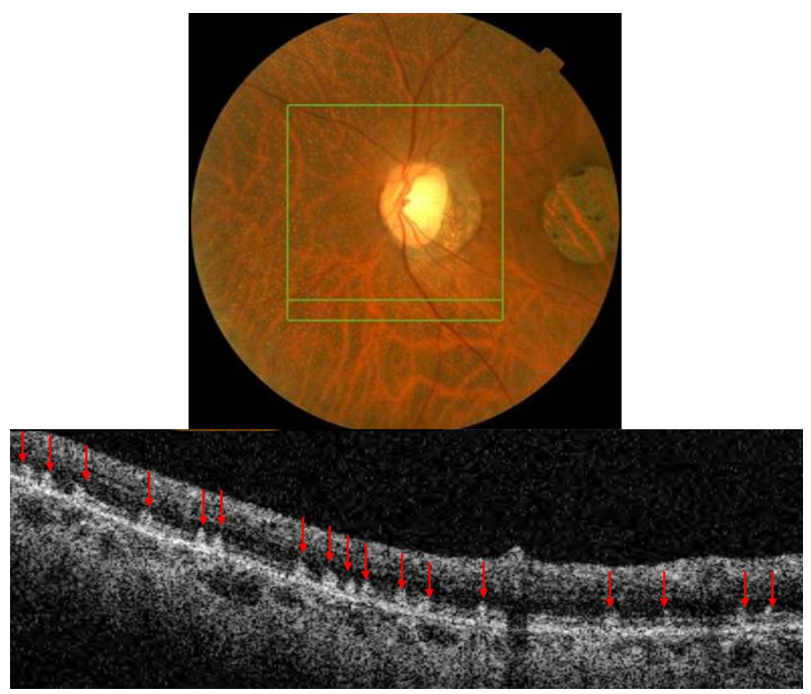

Figure 14 The B-scan line on the fundus photograph (top) has the same width as the B-scan SD-OCT image (bottom) demonstrating the appearance of white deposits (red arrows) in fundus albi.

Abbreviation: SD-OCT, spectral domain optical coherence tomography.

\section{B-scan SD-OCT reflectivity pattern}

SD-OCT imaging reveals numerous hyper-reflective dots (red arrows) larger than the thickness of the PIL that appear to straddle through the PIL, extending into the inner and outer photoreceptor layer (Figure 14). SD-OCT shows with great precision their location anterior to the RPE. All retinal layers, including the RPE, PIL, and external limiting membrane are present and grossly intact, resulting in good visual acuity unless CD is present concomitant with FA.

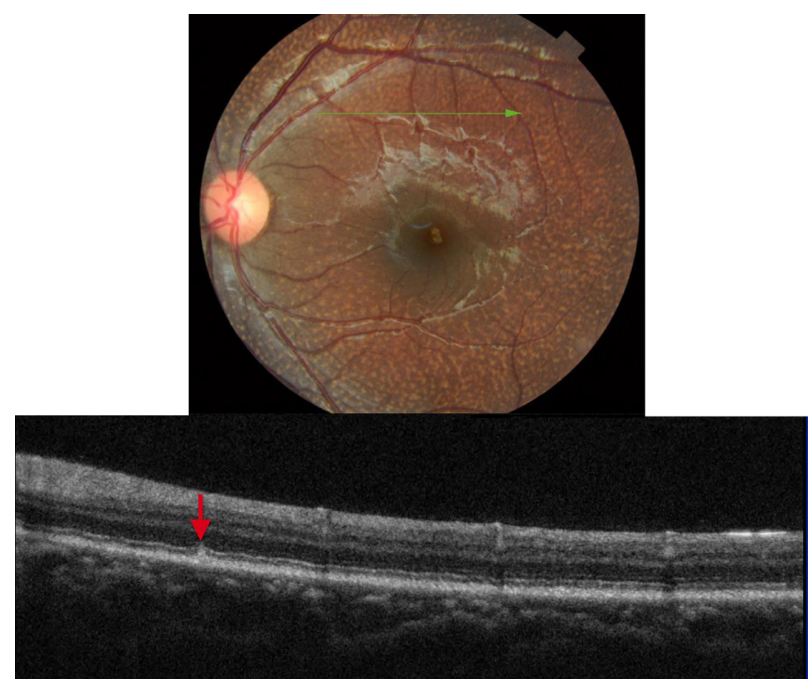

Figure 15 The B-scan line on the fundus photograph (top) has the same width as the B-scan SD-OCT image (bottom) demonstrating the appearance of early deposits in fundus albi (red arrow).

Abbreviation: SD-OCT, spectral domain optical coherence tomography.
Figure 15 shows SD-OCT image of a 6-year-old patient with FA. In comparison to the adult fundus shown in Figure 14, early FA appears to start as subretinal deposits between the PIL and the RPE layers (red arrow). The typical straddling through the PIL and extending into the inner and outer photoreceptor layer is likely to be associated with the longstanding FA deposition.

\section{RPE migration and RPE atrophy}

Fundus flavimaculatus (FF)/Stargardt disease

Stargardt disease is the most common juvenile macular dystrophy, accounting for approximately $7 \%$ of all juvenile macular dystrophies. ${ }^{28}$ It can present with or without white-yellow retinal deep lesions, that are called fundus flavimaculatus (FF). FF and Stargardt are variants of the same hereditary disease, sharing a similar genetic background..$^{28}$ They are both inherited as an autosomal recessive trait that is due to a mutation in the $A B C A 4$ gene. ${ }^{28}$ The composition of the deposits is presently unknown, and they exhibit variable patterns and shapes of hyper-reflective material. ${ }^{28,29}$ Clinically they appear as deep more-yellow-than-white ill-defined retinal lesions, commonly referred to as flecks in the literature. ${ }^{28,29}$ Even though they appear similar to drusen deposits, FF flecks are more likely to have fishtail, rather than round shapes (Figure 16). Flecks can be localized in the perifoveal region or can be scattered throughout the posterior pole and mid-periphery. ${ }^{28,29}$

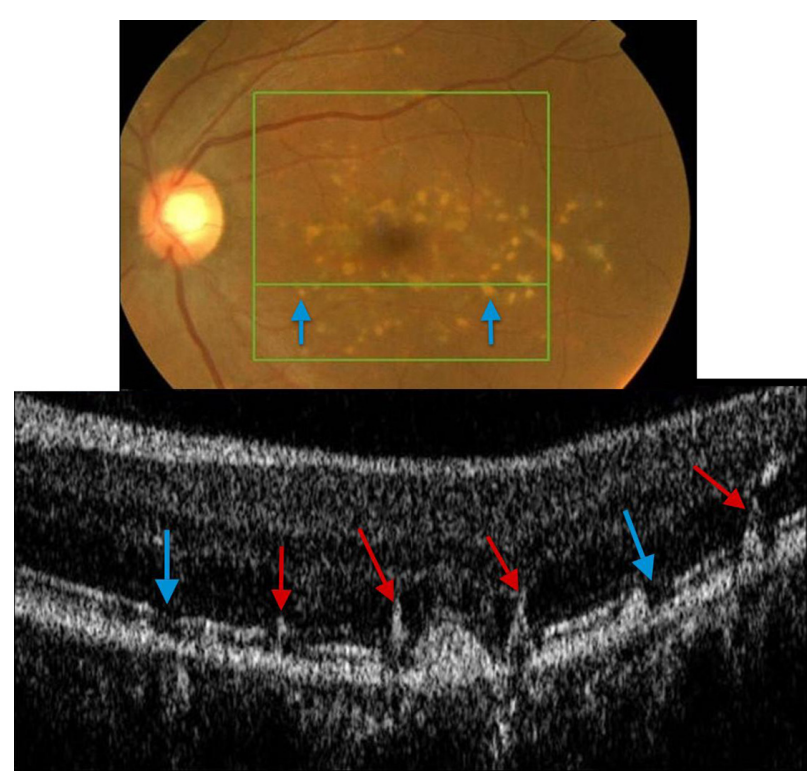

Figure 16 Fundus photograph (top) showing white FF flecks in the left eye of a patient with Stargardt disease; the B-scan line on the fundus photograph has the same width as the B-scan SD-OCT image (bottom) demonstrating areas of RPE migration (red arrows) and areas of RPE dropout (blue arrows).

Abbreviations: SD-OCT, spectral domain optical coherence tomography; FF, fundus flavimaculatus; RPE, retinal pigment epithelium. 


\section{B-scan SD-OCT reflectivity pattern}

FF flecks appear very different from drusen deposits. RPE pigment migration (red arrows) to the inner retina is clearly observed (Figure 16), leaving areas of RPE dropout (blue arrows) consistent with a yellow-white appearance clinically. Up to date, there are no histology reports available of the composition of FF flecks, likely due to the fact that they represent areas of RPE dropout.

\section{Bietti crystalline dystrophy (BCD)}

Bietti crystalline dystrophy is an autosomal recessive retinal dystrophy characterized by a triad of glistening intraretinal crystals, RPE atrophy and hypertrophy, which is also known as tapetoretinal degeneration and choroidal sclerosis. ${ }^{30}$ Crystal deposits have been reported to be composed mainly of cholesterol or cholesterol ester with complex lipid inclusions. ${ }^{31}$ Mutation in the CYP4V2 gene was found to be associated with $\mathrm{BCD}{ }^{31}$ This gene codes for a protein involved in fatty acid metabolism. ${ }^{31}$ According to the recent literature, $\mathrm{BCD}$ is associated with abnormally high levels of triglycerides and cholesterol storage in the body, though direct correlation to any specific systemic disease has not yet been established. ${ }^{31}$

Clinically, BCD is characterized by the presence of minute crystal deposits scattered along the posterior pole and mid-periphery, retinitis pigmentosa-like pigment clumping, and atrophic appearing retina with easily visualized choroidal vasculature, which is also known as choroidal sclerosis (Figure 18). The literature also reports the presence of yellowish crystals in multiple types of cells, including choroidal fibroblasts, corneal keratocytes, and conjunctival and skin fibroblasts. Anterior segment OCT reveals hyperreflective crystal deposits within the cornea at the limbus (Figure 17).

\section{B-scan SD-OCT reflectivity pattern}

Perhaps one of the most revealing characteristics of BCD is a complete absence of the PIL (Figure 18). A thin Bruch's layer can be easily visualized due to the extreme RPE thinning. Small highly-reflective dots are seen on the anterior surface of Bruch's membrane with more subtle hyperreflective dots scattered throughout inner retinal layers (blue arrows).

\section{Punctate inner choroidopathy (PIC)}

PIC is a part of the White Dot Syndromes family, which is a group of disorders characterized by multiple whitish inflammatory lesions located at the level of the outer retina

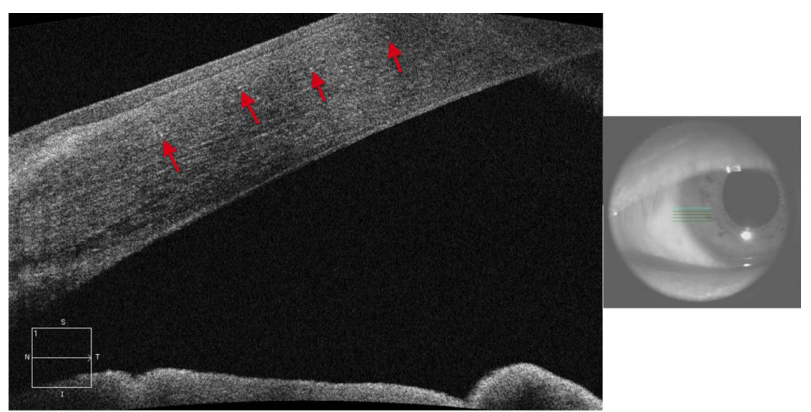

Figure 17 Anterior segment SD-OCT image demonstrating hyper-reflective crystals (red arrows) in the limbus area of the eye of a patient with Bietti crystalline dystrophy (BCD).

Abbreviation: SD-OCT, spectral domain optical coherence tomography.

and choroid. ${ }^{32,33}$ PIC normally affects young myopic women between the ages of 20 to 45 years of age. It presents as small multifocal well-circumscribed hypopigmented and pigmented fibrotic scars often located in both eyes (Figure 19). The color can be gray-white or yellow with a cuff of pigment at the level of the inner choroid and retinal pigment epithelium (RPE). It often resembles signs of ocular histoplasmosis (POHS) and unless an active vitritis is present, it is often difficult to distinguish between the two diseases. In this paper, we will show how SD-OCT imaging can be used to differentiate between the two entities. When active, PIC can be symptomatic with blurred vision up to 20/200 and complaints of photopsia.

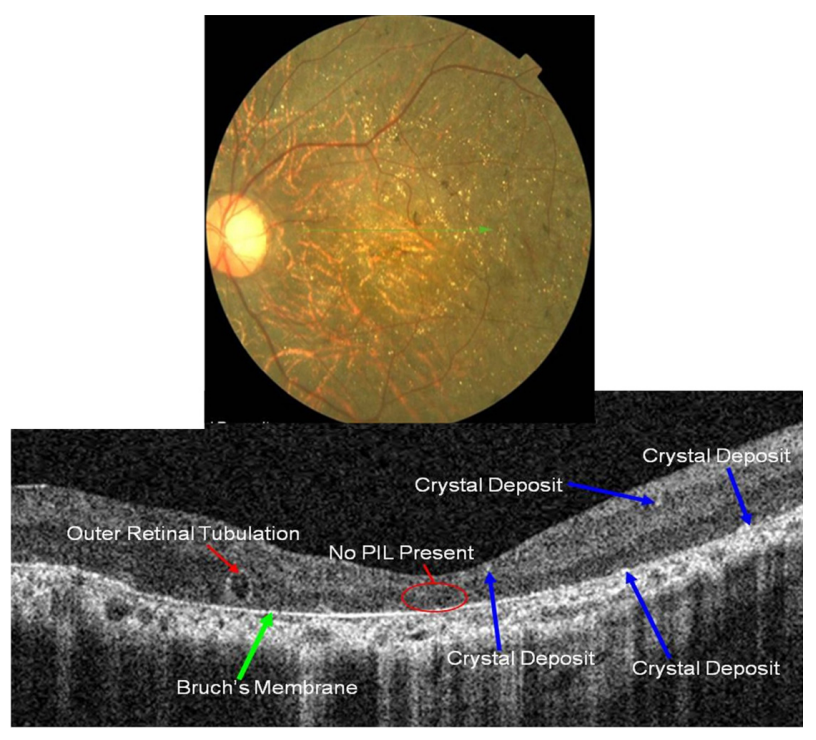

Figure 18 Fundus photograph (top) showing BCD triad, glistening crystal deposits, tapetoretinal degeneration and choroidal sclerosis, in the left eye of a patient with $\mathrm{BCD}$; the B-scan line on the fundus photograph has the same width as the B-scan SD-OCT image (bottom) demonstrating the appearance of crystalline deposits and the absence of PIL and RPE layers.

Abbreviations: BDC, Bietti crystalline dystrophy; SD-OCT, spectral domain optical coherence tomography. 


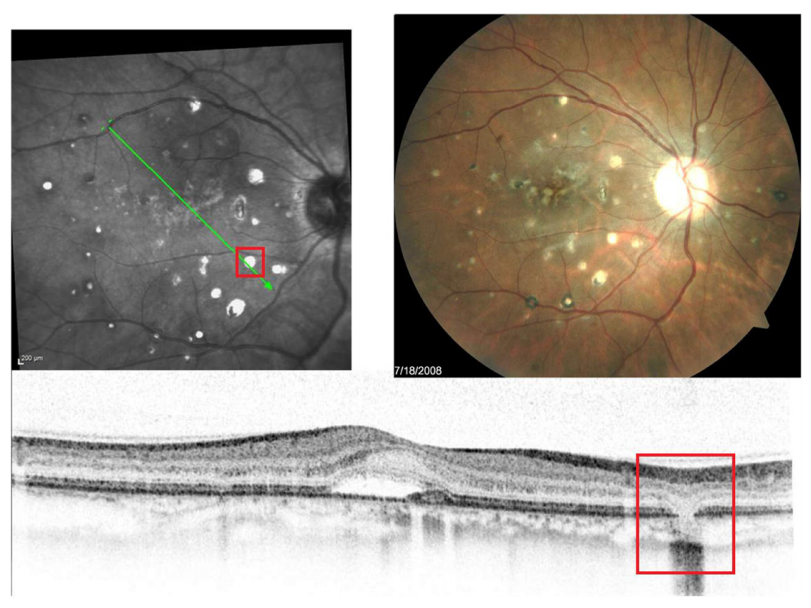

Figure 19 Fundus photographs (top) showing white lesions in the right eye of a patient with PIC; the B-scan line on the fundus photograph (top-left) has the same width as the B-scan SD-OCT image (bottom) demonstrating missing retinal layers in the area of the lesion.

Abbreviations: PIC, punctate inner choroidopathy; SD-OCT, spectral domain optical coherence tomography.

\section{B-scan SD-OCT reflectivity pattern}

SD-OCT shows a complete loss of all retinal layers at the level of white lesions (Figure 19). The RPE and PIL cannot be visualized, and even Bruch's membrane and choriocapillaris are missing in the area of the defect. This finding is virtually pathognomonic of PIC. The SD-OCT image of POHS, on the other hand, differs significantly from PIC (Figure 20), showing elevated rather than atrophic RPE and PIL layers.
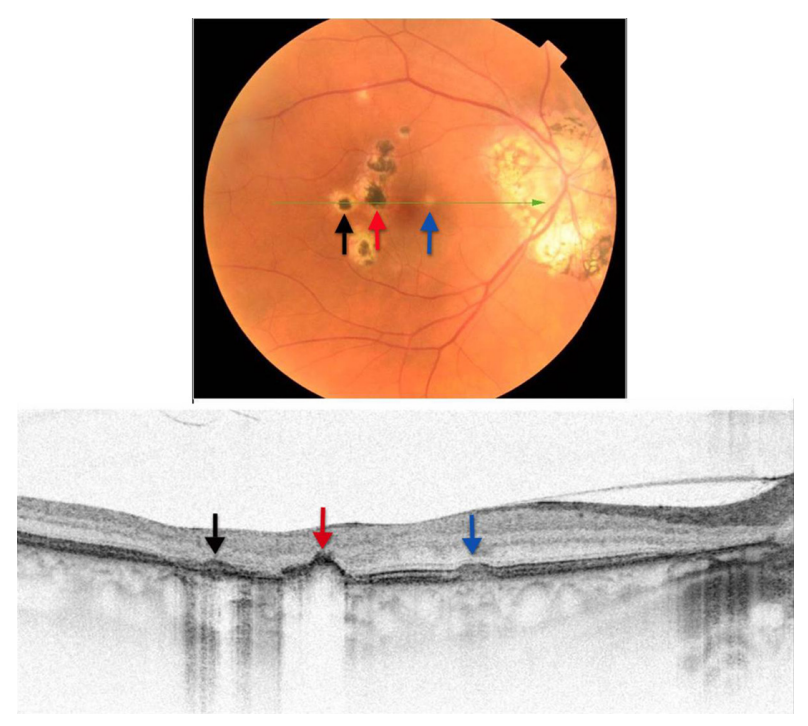

Figure 20 Fundus photograph (top) showing hypo- and hyper-pigmented lesions in the right eye of a patient with POHS; the B-scan line on the fundus photograph has the same width as the B-scan SD-OCT image (bottom) demonstrating the three different types of lesions: the area of pigment deposition surrounded by the area of RPE atrophy (black arrow), RPE migration (red arrow) and drusen-like apigmented pigment deposition (blue arrow).

Abbreviations: SD-OCT, spectral domain optical coherence tomography; RPE, retinal pigment epithelium; POHS, presumed ocular histoplasmosis.

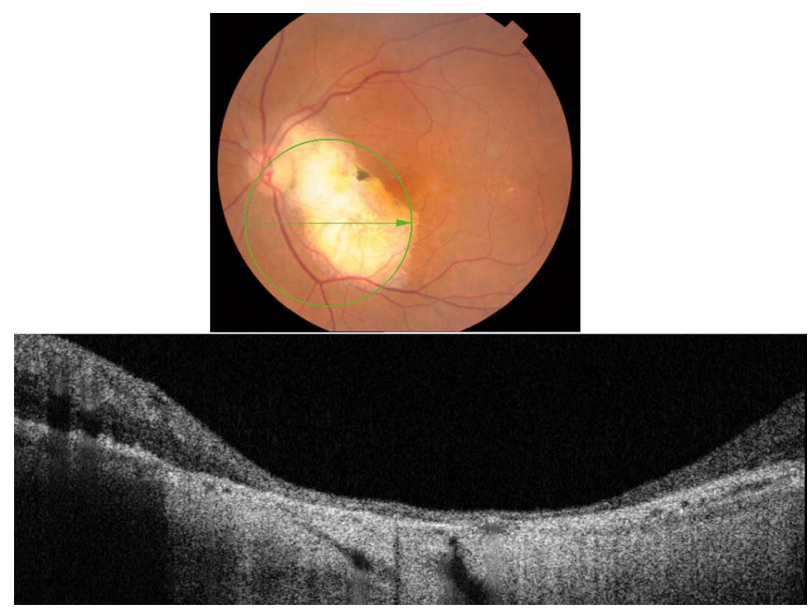

Figure 2 I The B-scan line on the fundus photograph (top) has the same width as the B-scan SD-OCT image (bottom) demonstrating the appearance of chorioretinal atrophy.

Abbreviation: SD-OCT, spectral domain optical coherence tomography.

Going from left to right, the B-scan through the three different types of chorioretinal lesions in POHS show the area of pigment deposition surrounded by the area of RPE atrophy (black arrow), RPE migration (red arrow), and drusen-like apigmented pigment deposition (blue arrow).

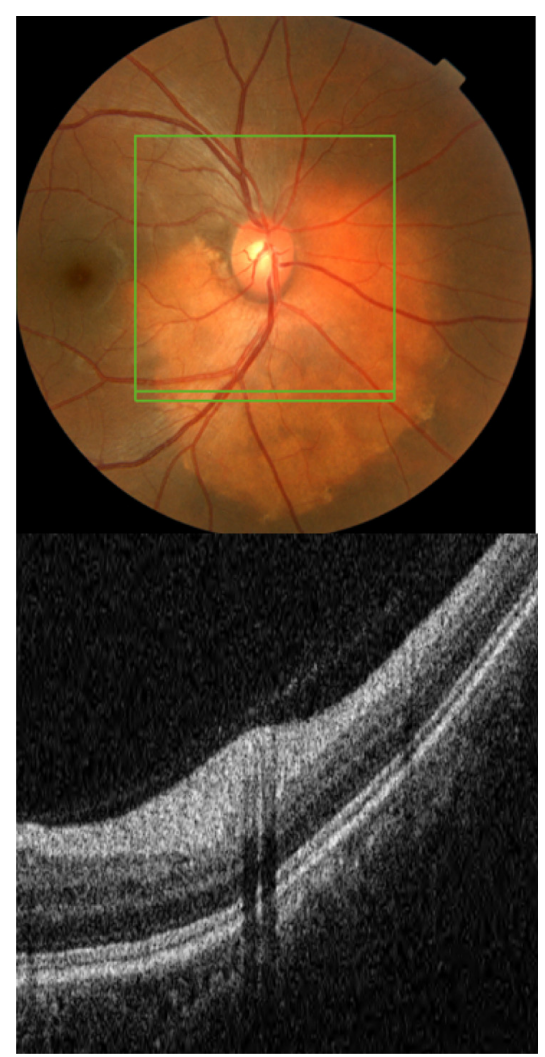

Figure 22 The B-scan line on the fundus photograph (top) has the same width as the B-scan SD-OCT image (bottom) demonstrating the appearance of osseous choristoma. Abbreviation: SD-OCT, spectral domain optical coherence tomography. 


\section{Chorioretinal scar}

Factored into the differential diagnosis of white retinal lesions is the atrophic chorioretinal scar. This case presents a patient who underwent sectoral pan-retinal photocoagulation (PRP) argon laser treatment of the left eye secondary to a polyploidal neovascular membrane. B-scan SD-OCT imaging revealed severe chorioretinal atrophy that resulted in complete absence of all retinal layers, including the RPE, PIL, and external limiting membrane (Figure 21).

\section{Osseous choristoma}

Osseous choristoma is defined as a congenital tumor consisting of ectopic osseous tissue in the choroid. ${ }^{34}$ It is more prevalent in Caucasians and is reported more frequently in women than men. It is composed of bony trabeculae with numerous osteocytes and occasional osteoclasts. ${ }^{34}$ Fundoscopically, osseous choristoma appears yellowish-white to sometimes reddish in color (Figure 22).${ }^{34}$ It can be elevated ranging from 0.5 to $2.00 \mathrm{~mm}$ elevation. ${ }^{35}$ The size ranges from $1.5 \times 2 \mathrm{DD}$ to $9 \times 15 \mathrm{DD} .{ }^{35}$ It has been reported that RPE can show areas of depigmentation and areas of RPE clumping; whereas, retina overlying the tumor can show focal degeneration of outer segments. ${ }^{34}$ Given the relatively benign and silent nature of the tumor, no treatment is usually recommended. However, argon laser treatment can be used if choroidal neovascularization develops. ${ }^{34}$

\section{B-scan SD-OCT reflectivity pattern}

As Figure 22 shows, SD-OCT imaging of osseous choristoma reveals intact RPE, PIL, and external limiting membrane layers. Areas of thickened retina can be observed; however, no mass under the retina and no retinal atrophy is associated with osseous choristoma.

\section{Conclusion}

Retinal spots, dots, and other white lesions can often present a diagnostic challenge for clinicians. The aforementioned cases, summarized in Table 1, demonstrate the importance of SD-OCT in the identification of the unique B-scan SD-OCT reflectivity patterns of the presented retinal abnormalities. SD-OCT is an ideal diagnostic tool to localize various deposits to a specific retinal layer, thus enhancing the differential diagnosis of retinal white dots and spots. Knowing how to interpret images of this remarkable technology can provide significant insight into characteristic features of these conditions often facilitating immediate diagnosis.

Table I Summary of B-scan SD-OCT reflectivity patterns of the presented dots, spots and other white retinal lesions

\begin{tabular}{|c|c|c|}
\hline Condition & SD-OCT reflectivity pattern & Associated figure \\
\hline \multicolumn{3}{|l|}{ Inner retinal lesions } \\
\hline MRNF & Hyper-reflective thickened RNFL & Figure 2 \\
\hline Cotton wool spots (CWS) & Nodule-shaped hyper-reflective thickened area within the RNFL & Figure 3 \\
\hline \multicolumn{3}{|l|}{ Outer retinal lesions } \\
\hline Hard exudates & Hyper-reflective minute dots at the level of OPL & Figure 4 \\
\hline Edema residues & Hyper-reflective deposits within the photoreceptor outer segment layer & Figure 8 \\
\hline \multicolumn{3}{|c|}{ RPE lesions/sub-RPE deposits } \\
\hline Cuticular hard drusen & $\begin{array}{l}\text { Discrete dome-shaped sub-RPE deposits; RPE is elevated; PIL is thinner, but present } \\
\text { and intact }\end{array}$ & Figure 9 \\
\hline Infiltrative hard drusen & Discrete sub-RPE deposits that infiltrate through the RPE and PIL; disrupted RPE and PIL & Figure 10 \\
\hline Subretinal drusenoid deposits & $\begin{array}{l}\text { Punctuate deposits localized between the RPE and the PIL layers; RPE is not elevated; } \\
\text { intact RPE and PIL }\end{array}$ & Figure II \\
\hline Soft drusen & $\begin{array}{l}\text { Sub-RPE deposits; RPE is largely elevated; compromised PIL above the deposits; } \\
\text { visualization of Bruch's membrane }\end{array}$ & Figure 12 \\
\hline Geographical atrophy & Localized absence of PIL and RPE layers; visualization of Bruch's membrane & Figure 13 \\
\hline Fundus albi & $\begin{array}{l}\text { Hyper-reflective deposits located anterior to RPE; straddling through the PIL; intact RPE } \\
\text { and PIL }\end{array}$ & Figure I4 \\
\hline \multicolumn{3}{|c|}{ RPE lesions/RPE migration and RPE atrophy } \\
\hline Fundus flavimaculatus & RPE migration, leaving areas of RPE dropout & Figure 16 \\
\hline Bietti crystalline dystrophy & $\begin{array}{l}\text { Hyper-reflective dots on top of Bruch's membrane; complete absence of PIL; } \\
\text { attenuated RPE; visualization of Bruch's membrane }\end{array}$ & Figure 18 \\
\hline PIC & Complete loss of all retinal layers, including Bruch's membrane, at the level of white lesions & Figure 19 \\
\hline POHS & Pigment deposition; RPE migration and atrophy & Figure 20 \\
\hline Post-PRP chorioretinal scar & RPE atrophy, absence of PIL, visualization of Bruch's membrane & Figure 21 \\
\hline Osseous choristoma & Thickened inner retina, intact RPE and PIL, no mass observed & Figure 22 \\
\hline
\end{tabular}

Abbreviations: MRNF, myelinated retinal nerve fiber layer; RNFL, retinal nerve fiber layer; OPL, outer plexiform layer; CWS, cotton wool spots; RPE, retinal pigment epithelium; PIL, photoreceptor integrity line; PIC, punctate inner choroidopathy; POHS, presumed ocular histoplasmosis syndrome; PRP, pan-retinal photocoagulation. 


\section{Disclosure}

The authors report no conflicts of interest in this work.

\section{References}

1. Chen TC, Cense B, Pierce MC, et al. Spectral domain optical coherence tomography: ultra-high speed, ultra-high resolution ophthalmic imaging. Arch Ophthalmol. 2005;123(12):1715-1720.

2. Alam S, Zawadzki RJ, Choi S, et al. Clinical application of rapid serial fourier-domain optical coherence tomography for macular imaging. Ophthalmology. 2006;113(8):1425-1431.

3. Podoleanu AG. Optical coherence tomography. Br J Radiol. 2005; 78(935):976-988.

4. Kim JS, Ishikawa H, Gabriele ML, et al. Retinal nerve fiber layer thickness measurement comparability between time domain optical coherence tomography (OCT) and spectral domain OCT. Invest Ophthalmol Vis Sci. 2010;51(2):896-902.

5. Xi P, Mei K, Bräuler T, Zhou C, Ren Q. Evaluation of spectrometric parameters in spectral-domain optical coherence tomography. Appl Opt. 2011;50(3):366-372.

6. Watanabe Y, Itagaki T. Real-time display on Fourier domain optical coherence tomography system using a graphics processing unit. J Biomed Opt. 2009;14(6):060506.

7. Srinivasan VJ, Adler DC, Chen Y, et al. Ultrahigh-speed optical coherence tomography for three-dimensional and en face imaging of the retina and optic nerve head. Invest Ophthalmol Vis Sci. 2008;49(11): 5103-5110.

8. Sarks SH, Arnold JK, Killingsworth MC, Sarks JP. Early drusen formation in the normal and aging eye and their relation to age related maculopathy; a clinicopathological study. Br J Ophthalmol. 1999; 83(3):358-368.

9. Wang M, Sander B, la Cour M, Larsen M. Clinical characteristics of subretinal deposits in central serous chorioretinopathy. Acta Ophthalmol Scand. 2005;83(6):691-696.

10. Sherman J, Yannuzzi LA, Madonna RJ, Nath S, Bababekova Y. The Photoreceptor Integrity Line: As Revealed by Spectral Domain OCT. New York: Lulu.com; 2008.

11. Litts K, Messinger J, Sloan K, Spaide R, Curcio C. Sources of reflectivity in optical coherence tomography (OCT) of retina: candidates near the innerouter segment (IS/OS) junction. Abstract 1322/A259.ARVO Conference; May 2, 2011; Fort Lauderdale, FL. Available at: http://www.abstractsonline.com/plan/ViewAbstract.aspx $? \mathrm{mID}=2684 \&$ sKey $=\mathrm{a} 9534887$ 8f0d-44c9-9838-df773b6c095b\&cKey=7f02ab5d-342b-430f-8$\mathrm{dc} 1-4 \mathrm{e} 2 \mathrm{bc} 2114 \mathrm{dbb} \& \mathrm{mKey}=\% 7 \mathrm{~B} 6 \mathrm{~F} 224 \mathrm{~A} 2 \mathrm{D}-\mathrm{AF} 6 \mathrm{~A}-4533-8 \mathrm{BBB}-$ 6A8D7B26EDB3\%7D. Accessed September 16, 2011.

12. Costa RA, Calucci D, Skaf M, et al. Optical coherence tomography 3 : automatic delineation of the outer neural retinal boundary and its influence on retinal thickness measurements. Invest Ophthalmol Vis Sci. 2004;45(7):2399-2406.

13. Ota M, Tsujikawa A, Murakami T, et al. Association between integrity of foveal photoreceptor layer and visual acuity in branch retinal vein occlusion. Br J Ophthalmol. 2007;91(12):1644-1649.

14. Shin HJ, Chung H, Kim HC. Association between integrity of foveal photoreceptor layer and visual outcome in retinal vein occlusion. Acta Ophthalmol. 2011;89(1):e35-e40.

Eye and Brain

\section{Publish your work in this journal}

Eye and Brain is an international, peer-reviewed, open access journal focusing on clinical and experimental research in the field of neuro-ophthalmology. All aspects of patient care are addressed within the journal as well as basic research. Papers covering original research, basic science, clinical and epidemiological studies, reviews and Submit your manuscript here: http://www.dovepress.com/eye-and-brain-journal
15. Tarabishy AB, Alexandrou TJ, Traboulsi EI. Syndrome of myelinated retinal nerve fibers, myopia, and amblyopia: a review. Surv Ophthalmol. 2007;52(6):588-596.

16. Arroyo JG. Cotton-wool spots may challenge diagnosis. Review of Opthalmology serial on the Internet: April, 2004 cited April 15, 2004. Available at: http://www.revophth.com/content/d/retinal_insider/ i/1332/c/25556/. Accessed April 10, 2011.

17. Gomez ML, Mojana F, Bartsch DU, Freeman WR. Imaging of longterm retinal damage after resolved cotton wool spots. Ophthalmology. 2009;116(12):2407-2414

18. Schmidt $D$. The mystery of cotton-wool spots - a review of recent and historical descriptions. Eur J Med Res. 2008;24;13(6):231-266.

19. McLeod D. Why cotton wool spots should not be regarded as retinal nerve fiber layer infarcts. Br J Ophthalmol. 2005;89(2):229-237.

20. Kanski JJ. Clinical Ophthalmology: A Systematic Approach. 5th ed. Philadelphia, PA: Butterworth Heinemann Elsevier; 2007

21. Wang M, Munch IC, Hasler PW, Prünte C, Larsen M. Central serous chorioretinopathy. Acta Ophthalmol. 2008;86(2):126-145.

22. Umeda S, Suzuki MT, Okamoto H, et al. Molecular composition of drusen and possible involvement of anti-retinal autoimmunity in two different forms of macular degeneration in cynomolgus monkey (Macaca fascicularis). FASEB J. 2005;19(12):1683-1685.

23. Johnson PT, Lewis GP, Talaga KC, et al. Drusen-associated degeneration in the retina. Invest Ophthalmol Vis Sci. 2003;44(10):4481-4488.

24. Spaide RF, Curcio CA. Drusen characterization with multimodal imaging. Retina. 2010;30(9):1441-1454.

25. Hirose E, Inoue Y, Morimura H, et al. Mutations in the 11-cis retinol dehydrogenase gene in Japanese patients with Fundus albipunctatus. Invest Ophthalmol Vis Sci. 2000;41(12):3933-3935.

26. Nakamura M, Hotta Y, Tanikawa A, Terasaki H, Miyake Y. A high association with cone dystrophy in Fundus albipunctatus caused by mutations of the RDH5 gene. Invest Ophthalmol Vis Sci. 2000;41(12):3925-3932.

27. Niwa Y, Kondo M, Ueno S, Nakamura M, Terasaki H, Miyake Y. Cone and rod dysfunction in fundus albipunctatus with RDH5 mutation: an electrophysiological study. Invest Ophthalmol Vis Sci. 2005; 46(4):1480-1485.

28. Ergun E, Hermann B, Wirtitsch M, et al. Assessment of central visual function in Stargardt's disease/fundus flavimaculatus with ultrahighresolution optical coherence tomography. Invest Ophthalmol Vis Sci. 2005;46(1):310-316.

29. Wirtitsch MG, Ergun E, Hermann B, et al. Ultrahigh resolution optical coherence tomography in macular dystrophy. Am J Ophthalmol. 2005; 140(6):976-983.

30. Drenser K, Sarraf D, Jain A, Small KW. Crystalline retinopathies. Surv Ophthalmol. 2006;51(6):535-549.

31. Li A, Jiao X, Munier FL, et al. Bietti crystalline corneoretinal dystrophy is caused by mutations in the novel gene CYP4V2. Am J Hum Genet. 2004;74(5):817-826.

32. Essex RW, Wong J, Fraser-Bell S, et al. Punctate inner choroidopathy: clinical features and outcomes. Arch Ophthalmol. 2010;128(8): 982-987.

33. Quillen DA, Davis JB, Gottlieb JL, et al. The white dot syndromes. Am J Ophthalmol. 2004;137(3):538-550.

34. Kim BH, Henderson BA. Intraocular choristoma. Semin Ophthalmol. 2005;20(4):223-229.

35. Gass JD. New observations concerning choroidal osteomas. Int Ophthalmol. 1979;1(2):71-84.

evaluations, guidelines, expert opinion and commentary, case reports and extended reports are welcome. The manuscript management system is completely online and includes a very quick and fair peer-review system, which is all easy to use. Visit http://www.dovepress.com/ testimonials.php to read real quotes from published authors. 